\title{
A heuristic algorithm for the circulation plan of railway electrical motor units
}

\author{
J. Miao ${ }^{1}$, Y. Yu ${ }^{2} \&$ Y. Wang ${ }^{1}$ \\ ${ }^{I}$ The State Key Laboratory of Rail Traffic Control and Safety, \\ Beijing Jiao University, China \\ ${ }^{2}$ Institute of Textiles and Clothing, \\ The Hong Kong Polytechnic University, Hong Kong
}

\begin{abstract}
The rolling stock rostering problem with maintenance constraints is NP-hard. In this paper, a two-stage approach is proposed. Relaxing the maintenance constraints, a transition network with minimum train unit is established in the first stage. A heuristic algorithm based on the transitions interchange is devised to search for a feasible maintenance route. The algorithm is verified with the operational timetable of Guangzhou-Shenzhen railway, and the performance is satisfactory. Without consideration of empty movements, the algorithm can just be applied in the situation where the depots are well planned.
\end{abstract}

Keywords: electrical motor units, circulation plan, heuristic algorithm.

\section{Introduction}

Before the year 2007, all the passenger trains on the traditional railway lines in China were built-up with locomotive(s) and cars. The locomotives and passenger car fleets are managed under a different department and operated separately according to different plans. As the passenger trains often run across great distances and take several days, most of the trains are allocated with multiple car fleets. A car fleet is often assigned to execute a given train, except for a few short-distance trains. The composition of fleets often persists over a relatively long time unless there is maintenance.

With the train speed upgrade project and the development and operation of passenger dedicated rail lines, Electrical Motor Units (EMU) are employed in the operation of high-speed trains which run at $200-300 \mathrm{~km} / \mathrm{h}$ speed. There are two 
component forms of EMU in railway of China: the short form with 8 vehicles as a unit and the long form with 16 vehicles as a unit. Two short units can be coupled together if they are of the same model. The combination and disassembling of EMU are conducted in the depot. Inspections and maintenances on the EMU must be performed based on the regulated operational time and distance [1].

Due to the high cost of EMU, and the fact that most of the high-speed trains are operated in short and middle-range distance, shorter non-commercial time is desired to improve the utilization of EMU. As a result, one EMU is often required to operate for multiple trains.

Based on the characteristics of the operation of EMU in railway of China, the optimization algorithm of EMU circulation problem with given train schedule is studied in this paper. The rest of the paper is organized as the following: an elaboration to the circulation of EMU in railway of China is presented in Section 2 , the literature review is given in Section 3, the proposed heuristic algorithm is investigated in Section 4, the results of our computational experiments are presented in Section 5, and we conclude in Section 6.

\section{The EMU circulation problem in railway of China}

Several terminologies from [2] are adopted in our discussion of the EMU circulation problem. A route is a path between two given stations. A ride is a train unit on a certain route with distinct departure and arrival time. The connection relationship of two consecutive rides carried out by one EMU is called transition.

In China's railway, the type of EMU and the number of cars are assigned during the line plan stage. Hence, only the transition between the rides carried out by same type of EMU are to be investigated, and no shunting will occur in stations.

We define a rotation is the order of rides performed by one EMU from the end of a class one maintenance to the end of the next maintenance. A circulation is the aggregation of all rotations. A feasible circulation in China railway must meet the following constraints:

(1) Every train must be assigned with required type and amount of EMU. i.e., every scheduled ride must belong to one single rotation. This is called the ride cover constraint.

(2) The transition time should be longer than the necessary turnaround time in stations.

(3) To meet the maintenance regulation of EMU. In railway of China, the $1^{\text {st }}$ class and the $2^{\text {nd }}$ class inspection and maintenance can be carried out in any depot. The $1^{\text {st }}$ class inspections are daily inspections with a period of $4000 \mathrm{~km}$ or $48 \mathrm{~h}$ for the most types of EMU. The $2^{\text {nd }}$ and higher class inspections or maintenance are generally with long intervals and are time-consuming, and such inspections are arranged by dispatcher according to the short term operation plan, and are not studied in our research. 
Typically, to meet the demand of passenger flow, unless absolute necessary, there will be no empty train movements in China in order to reduce the waste of the infrastructure capacity. If the feasible EMU circulation for a timetable does not exist, the operator will reschedule the timetable with changing the departure time, canceling or adding some trains till the feasible solution emergence. Therefore, the empty movement is not included in our work.

Without the empty movements, indexes for evaluating circulation are the amount of EMU, and the number of inspections.

(1) Amount of EMU

Because the amount of EMU is less till now in China, to use as fewer as possible EMU to carry out scheduled trains is the top objective of the railway operator. For a given timetable, the number of EMU needed can be calculated by:

$$
\text { number of EMU }=\frac{\sum \text { travel time }+\min \sum \text { transition time }}{\text { timetable' } \text { stime horizon }}
$$

(2) Number of inspections

Less number of inspections is better as long as the regulations are met. For a given timetable, there are time and mileage constraints to determine the number of inspection, and the lower bound can be calculated by the following respectively:

$$
\begin{gathered}
\text { number of inspection by time }=\frac{\text { amount of EMU } \times \text { timetable' stime horizon }}{\text { timeinterval for inspection }} \\
\text { number of inspection by mile }=\frac{\sum \text { travel mileage }}{\text { mileage for inspection }}
\end{gathered}
$$

Hence, the lower bound of inspection times is determined by:

minimum number of inspection $=$

$\max (\lceil$ number of inspection by time $\rceil,\lceil$ number of inspection by mile $\rceil)$

The increase of number of inspection renders higher operation cost and consumes more depot capacity.

Based on the above discussion, we can draw a conclusion that EMU circulation problem is a Rolling Stock Rostering problem with time and distance maintenance constraints (RSR-M).

\section{Literature review}

Lots of research has been conducted on the rolling stock circulation. In Anderegg et al. [2], some basic concept in the operation of passenger trains are introduced, Erlebach et al. [3] summarizes the operation of passenger trains into basic models and variable constraints, and he states that the Rolling Stock Rostering with Maintenance constraint is a NP-Hard problem.

In the literature, there are different ways in solving such problems with different constraints and characteristics due to the background of the problems and the empirical demands. Arianna et al. [4] studies the circulation planning of multiple types train unit operated on a single line. The objective of their research 
is to minimize the number of train units and the operational kilometers under the constraints that the given amount of passenger being matched with the consideration of the sequence of train units. Mixed Integer Programming is utilized and CPLEX is employed in solving the problem. Fioole et al. [5] and Maróti [6] optimize the rolling stock circulation with the consideration of trains combining and splitting. CPLEX is employed in the solution, and several heuristic methods are designed to improve the efficiency. Marc and Kroon [7] models the rolling stock circulation problem with Integer Programming. The objective of the model is to maximize the satisfaction of passenger's demand, and the combining and splitting of train units are also taken into consideration. The Column Generation is used in solving the model. Cordeau et al. [8] studies the simultaneous optimal problem of the locomotives and cars. A basic model is built for the assignment of locomotives and cars, and the model is extended with maintenance constraints. Column generation methods are embedded into the Brunch and bound algorithm and this algorithm is used to solve the mentioned problem. Zhao [9] studies the daily circulation of train units, the problem is broken into two parts, one is the train assignment and the other is the maintenance routing. The problem is modeled with the Traveling Salesman Problem (TSP) and a local searching algorithm based on probability theory is designed to find the solution.

Our study is different from the previous ones including in two things. First, we hierarchize the multi-objective by constructing the transition network with minimum train unit number and heuristic searching maintenance routes. Second, an interchange method with heuristic is adopted to find the feasible solution.

\section{The heuristic algorithm for the EMU circulation}

The circulation problem is with great complexity due to the ride cover constraint and maintenance constraint, and it is proved to be a NP-Hard problem in $[3,10]$. A review on circulation planning algorithms is found in [11], where algorithms like set partition with path generation, TSP heuristic with random choice or Lagrange relax, min-cost flow or bipartite matching with heuristic searching are discussed.

In our proposed algorithm, an interchange method similar to $[2,10,11]$ is adopted. But with improvement on the network construction and the heuristic rules. A two-stage scheme is employed in our algorithm. The objective of the first stage is to minimize the amount of EMU and interchange method is applied in the construction of the transition network without the maintenance constraints. In the second stage, the interchange method is applied in finding the route that meet the maintenance constraint in the network constructed during the first stage, and the final circulation plan is completed at the end of the second stage. There are two steps in the second stage: (a) Finding of maintenance route, the maintenance route may violate the maintenance constraint. (b) Adjustment of maintenance route, so that it meets the maintenance constraint, and the solution of the EMU circulation is thereby formed.

In the following, we will discuss the construction of transition network in section 4.1, and the construction of maintenance route will be introduced in 
section 4.2 , in section 4.3 , the process of adjustment of maintenance routes to make it a legal circulation is presented.

\subsection{Construction of the transition network}

For a given timetable, some notions are defined in table 1.

Table 1: $\quad$ Notions definition.

\begin{tabular}{ll}
\hline symbol & \multicolumn{1}{c}{ meaning } \\
\hline$\tau$ & the time horizon of a timetable, it's one day for China railway \\
$S$ & the set of the stations where a ride is begin or end \\
$S^{c}$ & the set of the stations which connect the depot directly \\
$b_{s}$ & the minimum station turnaround time \\
$\varphi$ & the minimum duration for the class one maintenance \\
$R$ & the set of the rides in the time horizon \\
$r_{i}$ & the $i^{\text {th }}$ ride in the set $R, i=1, \ldots, n$ \\
$r_{i}^{d}$ & the departure time of $r_{i}$ \\
$r_{i}^{a}$ & the arrival time of $r_{i}$ \\
$r_{i}^{d s}$ & the departure station of $r_{i}$ \\
$r_{i}^{a s}$ & the arrival station of $r_{i}$ \\
$r_{i}^{m}$ & the travel mile of $r_{i}$ \\
$r_{i}^{j t}$ & the travel time of $r_{i}$ \\
$k$ & the minimum maintenance times \\
\hline
\end{tabular}

a station $s \in S^{c}$ is called maintenance station. Maintenance is performed only at a maintenance station.

A network $G(V, E, W)$, shown in Fig. 1, can be used to model the scheduled rides. Each node that belongs to the set $\mathrm{V}$ represents one ride, so the set $V=R$. The transition $e_{i j} \in E$, which indicates the ride $j$ is carried out by the EMU arrived as the ride $i$, is an arc in the $G . w_{i j} \in W$ represents the weight of $e_{i j}$ and its value is determined by the equation (5).

$$
w_{i j}=\left\{\begin{array}{cc}
r_{j}^{d}-r_{i}^{a} & r_{j}^{d s}=r_{i}^{a s} \wedge r_{j}^{d}-r_{i}^{a} \geq b_{r_{j}^{d s}} \\
\tau+r_{j}^{d}-r_{i}^{a} & r_{j}^{d s}=r_{i}^{a s} \wedge r_{j}^{d}-r_{i}^{a}<b_{r_{j}^{d s}} \\
+\infty & r_{j}^{d s} \neq r_{i}^{a s}
\end{array}\right.
$$

if $e_{i j}$ satisfies the following criterion,

$$
r_{j}^{d s} \in S^{c} \wedge \varphi \leq w_{i j}<2 \tau
$$

then $e_{i j}$ is a maintenance arc noted as $\bar{e}_{i j}$. 


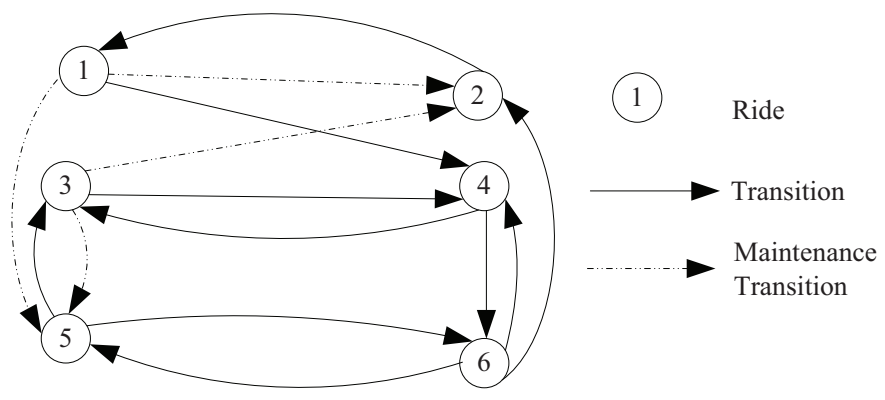

Figure 1: $\quad$ Transition network.

In the network, a rotation is a route which starts from a head node of maintenance arc, and ends up with a tail node of maintenance arc. Any node must be covered by only one route.

The first step in the construction of the ride network is to establish the connection relations between nodes. Although the EMU of a terminated train can execute any departure train which meets the criterion of (5), if the total transition time cannot be guaranteed to be the minimum, the amount of EMU will be increased. Bipartite graph matching can be used to construct transition network with the objective of minimum total connecting time. The bipartite graph can be established with two sets of departure time and arrival time, and the matching weights from equation (5). To ensure perfect matching of bipartite graph, i.e. there is connection for every ride, for stations where the number of arrival trains and departure trains is not equal, empty movements of EMU must be added as compensation, i.e. to add some extra nodes so that the number of nodes in the two sets are equal.

There are many methods for solving such matching problems. In [12], First Arrival First Departure (FAFD) method is presented and it is proved to be able to achieve the optimal resolution, with the computational complexity of $O(n \log n)$. The assignment problem of EMU and the assignment problem of locomotives are identical when there is no coupling and uncoupling of trains, and the FAFD method can be employed to find optimal solution, noted as $\mathcal{Z}$.

A transition network must include as many as possible optimal matching solutions so that to supply a sufficient searching space. As the timetable of trains cycles with period, as indicated in [13], for any station, if the connection relations of $e_{i j}$ and $e_{i j}^{\prime}$ meets the criterion in (7), a new matching solution can be obtained by swapping the two connection relations of a given matching solution. This feature is illustrated in Fig. 2. Multiple optimal matching solutions can be derived from an initial solution $\mathcal{Z}$ according to (7).

$$
r_{j}^{d}-r_{i}^{a} \geq b_{s} \wedge \quad r_{j}^{d}-r_{i}^{a} \geq b_{s}, \quad \forall r_{j}^{d s}=r_{i}^{a s}, \forall r_{j}^{d s}=r_{i}^{a s}
$$




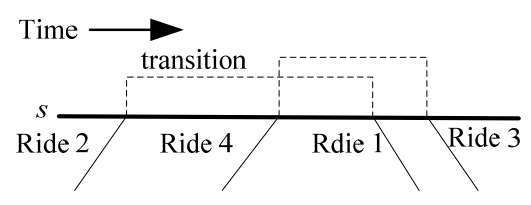

(a) initial transitions

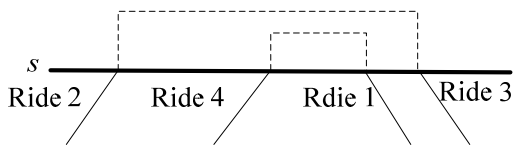

(b) transitions after interchanging

Figure 2: Transitions interchange.

The following notations are given for the definition of the transition network. For a given solution $\mathcal{Z}, k^{\mathcal{Z}}$ is the number of maintenance arcs, and $k^{*}=\max \left(k^{\mathcal{Z}}\right)$ for all $\mathcal{Z}$. For $\forall v \in V, \delta^{-}(v)$ is the times that node $v$ being the tail node of maintenance arc. The algorithm for the establishment of transition network is depicted in Fig. 3.

\section{Proc Establish_Netwok Begin}

Employ FAFD method to match rides with the objective of $\min \sum_{i=1}^{n} \sum_{j=1}^{n} w_{i j}$, and the result set $\mathcal{Z}$ is added to set $E$, let $k^{*}:=k^{\mathcal{Z}}, \delta^{-}(v)$ is set

$$
\text { according to } \mathcal{Z} \text {. }
$$

\section{Loop Begin}

If $\exists e_{i j} \in \mathcal{Z}, e_{i j} \in \mathcal{Z}$ meet the criteria in (6), where $e_{i j^{\prime}} \notin E$ or $e_{i j} \notin E$

\section{Then}

Swap $e_{i j}$ and $e_{i j}$ to have $\mathcal{Z}^{\prime}$, and the new path is added to $E$;

Update $\delta^{-}(v)$ and $k^{*}$, and let $\mathcal{Z}:=\mathcal{Z}^{\prime}$.

Else break loop

\section{Loop End}

Proc End

Figure 3: The establishment process of the transition network.

The rule for updating $\delta^{-}(v)$ is: if $k^{\mathcal{z}} \geq k$ and $v$ is the tail node in a maintenance arc, $\delta^{-}(v)$ will be increased by 1 .

If $k^{*}<k$ for the established network, there will be no solution for it.

\subsection{Generating the maintenance routes}

The objective of this process is to find at most $k^{*}$ routes in $G$, and these routes covers all nodes in the network, and each node are covered only once. Every route, which starts with the head node (included) of maintenance arc and ends 
with the head node (excluded) of another (or same, if the route is a circuit) maintenance arc, is called a maintenance route. A maintenance route may violate the maintenance constraint and it's the intermediate process of legal EMU circulation.

The duration of the maintenance route $p$, denoted by $p^{t}$, is the summation of travel time of all rides and all transition time included in the route, and the distance of the route, presented as $p^{m}$, is the total travel miles of the rides belonging to the route. Constants $\varepsilon$ and $\gamma$ are the maximum distance and time constraints for class one maintenance respectively, if $\eta_{p}=\max \left(p^{t} / \gamma, p^{m} / \varepsilon\right)$ is noted as the distance factor for route $p$, a circulation for an EMU is a maintenance route with $\eta \leq 1.0$.

A matching solution $\mathcal{Z}$ is consisted of one or more circuits that cover all nodes, the maintenance arcs split it into several routes. Equation (4) gives the lower bound of the optimal number of maintenance in an EMU circulation, i.e. the minimum number of routes. Hence, to get the optimal number of maintenance, $k$ non-mutually exclusive maintenance arcs should be selected as the starting and ending of a circulation. These maintenance arcs are the candidate maintenance arcs, noted as $\mathcal{C}$.

The first problem in searching for maintenance routes is the choice of $\mathcal{C}$. Every combination of maintenance arcs represents a group of $\mathcal{Z}$. There are cases where there are feasible solutions in the network, but they are not in the selected group of $\mathcal{Z}$. $\mathcal{C}$ must be changed and reselected for these cases.

To reduce the number of iterations, maintenance arcs with more matching solutions is more preferable as $\mathcal{C}$. The selection process is: first, select $\zeta$ nodes randomly as the candidate tail nodes of maintenance arcs, nodes with bigger $\delta^{-}(v)$ are with high priority, $\zeta$ maintenance arcs can be obtained based on these nodes. To speedup the searching for optimal solution, $\zeta$ is initialized as $\zeta=k$. Secondly, with the $\zeta$ maintenance arcs as known matching, the FAFD method is employed again to search for $\mathcal{Z}$. If the total weight of $\mathcal{Z}$ is bigger than the optimal matching weight, the nodes will be reselected, otherwise $\mathcal{Z}$ is the set of all maintenance routes.

\subsection{Adjustment of maintenance routes}

The maintenance routes have to be transformed into feasible solutions if they are non-feasible. Swapping connection relations is adopted to transform the solutions, the objective of the swapping is to make $0<\eta \leq 1$.

If the maintenance routes set is $P$, for two maintenance routes $\alpha \in P$ and $\beta \in P, v_{u}^{\alpha} \in V$ is the $u^{\text {th }}$ node in $\alpha, v_{l}^{\beta} \in V$ is the $l^{\text {th }}$ node in $\beta$, if there is 


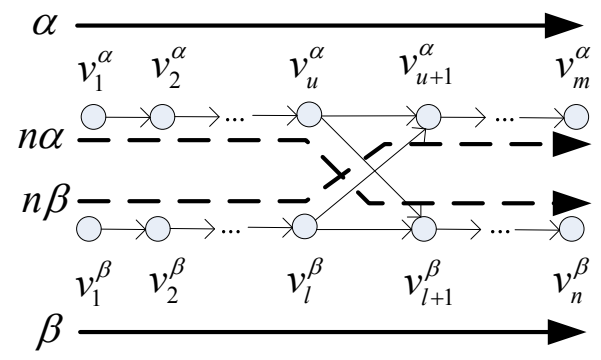

Figure 4: Swapping the connection to adjust maintenance routes.

$v^{\beta}{ }_{l+1} \in N^{+}\left(v_{u}^{\alpha}\right)$ and $v_{u+1}^{a} \in N^{+}\left(v_{l}^{\beta}\right)$, where $N^{+}\left(v_{u}^{\alpha}\right) \subseteq V$ are predecessor set of $v_{u}^{\alpha}$, then $\alpha$ intersects $\beta$ at $x$. Maintenance routes $\alpha$ and $\beta$ are interchangeable at $x$ to form new maintenance routes $n \alpha, n \beta$, this process is illustrated in Fig. 4 .

The objective of swapping is to shorten those long maintenance routes to make them feasible rotations. Therefore, the swapping should be performed on the longest illegal rotations in the rotation set, the point of intersection is preferably located in the middle of maintenance route so that to speedup this process. To avoid duplicated swapping at the same intersection point, an "escape-list" is used, and all the intersection points where the swapping is performed are listed in. Points in the list are not reused for swapping except for a better optimal status achieved at the point.

Let $\theta=\sum_{\forall \eta_{p}>1, p \in P}\left(\eta_{p}-1\right)$ be the optimal status of the set $P$, and is initialized as $\theta=+\infty$. $O$ is the global escape-list, and its minimum length is $|E|$. The algorithm for the adjustment of maintenance routes is given in Fig. 5 .

\section{Proc Adjust_MR Begin}

\section{Loop Begin}

Search for $\hat{p}$ with the maximum $\eta$ in $P$

If $\eta_{\hat{p}} \leq 1$ Then End Proc with $P$

Search for $\hat{x}$ in $\hat{p}$;

Swap maintenance routes at $\hat{x}$, and $\alpha, \beta$ are substituted by $n \alpha, n \beta$;

compute $\theta^{\prime}$, if $\theta^{\prime}<\theta$, then $\theta:=\theta^{\prime}$;

add $\hat{x}$ to $O$;

\section{Loop End}

\section{Proc End}

Figure 5: $\quad$ Procedure of adjusting the maintenance routes.

There will be no feasible solution in this candidate maintenance arcs set when a no-solution prompt returns from the algorithm. The algorithm in Section 4.2 
will be applied to update $\mathcal{C}$, and readjustment will occur under this condition. $\zeta$ will be increased by 1 if it is unable to update $\mathcal{C}$ with $\zeta$ until $\zeta=k^{*}$.

As the transition network is constructed with the shortest transition time, and this guaranteed minimum amount of EMU. The algorithm for the adjustment of maintenance route shortens the length of rotation rapidly. Meanwhile, the selection of maintenance arcs enables the fast finding of high quality solution. Consequently, our algorithm is fast in search of solutions with minimum EMU.

\subsection{Computational experiments}

The case study of algorithm is base on the actual timetable form GuangzhouShenzhen railway line which is located in the south China. There are 156 trains scheduled and 3 turnaround stations, which are Guangzhou, Guangzhou East and Shenzhen. The maintenance station is Guangzhou East. The total rides kilometrage is $20,368 \mathrm{Km}$ and the summation of travel time is 9,455 minutes. In the case study, we set the parameters $b_{s}=12, \varepsilon=4,000 \mathrm{Km}, \gamma=2,880 \mathrm{~min}$ and $\varphi=120 \mathrm{~min}$. According to equation (1) and (4), the minimum number of EMU utilized and the number of inspection is 13 and 7 respectively.

The transition network with $|E|=156,|V|=388$ and $k^{*}=8$ is established by 170 times interchange. The algorithm runs 10 rounds on the PC (Pentium4 3.0GHz, 512M Ram, Windows XP) and the results is listed in table 1. According to the different candidate maintenance arcs selected, the algorithm has various interchange iterations.

Table 2: $\quad$ Table of results.

\begin{tabular}{|l|c|c|c|c|}
\hline round & $\begin{array}{c}\text { Number of } \\
\text { Inspections }\end{array}$ & update times of $\mathcal{C}$ & $\begin{array}{c}\text { Total } \\
\text { interchange } \\
\text { times }\end{array}$ & $\begin{array}{c}\text { Runtime } \\
\text { (sec.) }\end{array}$ \\
\hline 1 & 7 & 1 & 7 & 0.078 \\
\hline 2 & 7 & 1 & 41 & 0.125 \\
\hline 3 & 7 & 1 & 22 & 0.063 \\
\hline 4 & 7 & 1 & 5 & 0.047 \\
\hline 5 & 7 & 1 & 18 & 0.078 \\
\hline 6 & 7 & 1 & 33 & 0.165 \\
\hline 7 & 7 & 1 & 3 & 0.063 \\
\hline 8 & 7 & 1 & 4 & 0.062 \\
\hline 9 & 7 & 1 & 15 & 0.089 \\
\hline 10 & 7 & 1 & 22 & 0.062 \\
\hline
\end{tabular}

\section{Conclusion}

The RSR problem imposes maintenance constraints on a matching problem, and this makes it a NP-hard problem. In this paper, a network approach for the EMU 
assignment is proposed, with the aim of minimizing the amount of EMU for operation so that to alleviate the shortage of EMU situation of railway of China. The network is divided into multiple searching spaces based on the possibility of the existence of high-quality solutions, and a heuristic algorithm is devised to search for feasible solutions in the searching spaces with a swapping method. The algorithm is verified with the operational time table of Guang-Shen railway.

Our algorithm is based on the assumption that the depots of EMU are wellplanned. There could be no solution when this assumption is not valid, and the algorithm has a low efficiency in the situation of no solution. Hence, to determine the existence of feasible solution in a shorter time and to find a way of dealing with the situation of no solution so as to guarantee the minimum of the amount of EMU is the direction of our future works.

\section{Acknowledgements}

This paper is based on the work carried out under the railway operation optimizing project (RCS2008ZZ003) which is supported by the state key laboratory of rail traffic control \& safety fund.

\section{References}

[1] The Ministry of Railway P.R.C., Temporary Rules for Railway Electrical Motor Units Operation \& maintenance, China Railway Publishing House, Beijing, pp 2-4, 2007.

[2] Anderegg, L., Eidenbenz, S., Gantenbein, M., Stamm, Ch., Taylor, D.S., Weber, B., Widmayer, P., Train Routing Algorithms: Concepts, Design, Choices, and Practical Considerations, Proc. of the $5^{\text {th }}$ workshop on algorithm engineering and experiments (ALENEX03), Baltimore, 2003.

[3] Erlebach T, Gantenbein M, Hürlimann D, Neyer G, Pagourtzis A, Penna $\mathrm{P}$, et al. On the complexity of train assignment problems. Proc. of ISAAC'01, International Symposium on Algorithms and Computation, Christchurch, 2001.

[4] Arianna A., Groot R., Kroon L., Schrihver A., Efficient circulation of railway rolling stock,. Transportation Science, 40(3), pp. 378-391, 2006.

[5] Fioole P.J, Kroon L., Maróti G., Schrijver A., A rolling stock circulation model for combining and splitting of passenger trains. European Journal of Operational Research, 174(2), pp. 1281-1297, 2006.

[6] Maróti G. Operations research models for railway rolling stock planning, $\mathrm{PhD}$ thesis, Technische Universiteit Eindhoven, 2006.

[7] Marc P., Kroon L., Circulation of railway rolling stock a branch-and-price approach. Computers \& Operational Research, 35(2), pp. 538-556, 2008.

[8] Cordeau J.F., Desaulniers G., Lingaya N., Soumis F., Desrosiers J., Simultaneous locomotive and car assignment at VIA Rail Canada. Transportation Research Part B, 35(8), pp. 767-787, 2001.

[9] Zhao P, Tomii N, Fukumura PN, Sakaguchi T. An algorithm for train-set scheduling based on probabilistic local search. Computers in Railways 
VIII, eds. J. Allan, R.J. Hill, C. A. Brebbia, G. Sciutto, S. Sone, WIT Press: Southampton, pp. 817-826, 2002

[10] Maróti G., Kroon L., Maintenance routing for Train units: The interchange model, Computer \& Operations Research, 34(4), pp. 11211140, 2007.

[11] Hong S P, Kim K M, Lee K Park B H, A pragmatic algorithm for the train-set routing: The case of Korea high-speed railway, Omega, 37 (3), pp. 637-645, 2009.

[12] Xiao L. W., Computerized Planning of the Optimal Locomotive Working Diagram, Journal of Changsha Railway University (In Chinese), 17(1), pp. 52-57, 1999.

[13] LI Zhizhong, SUN Yan, Optimize the Locomotive Working Diagram by Computer. Railway Transport and Economy (In Chinese), 5, pp. 12-15, 1988. 